\title{
SUPERSYMMETRIC APPROACH FOR GENERATING QUASI-EXACTLY SOLVABLE PERIODIC POTENTIALS
}

\author{
V. M. Tkachuk, O. Voznyak \\ Ivan Franko National University of Lviv, Department for Theoretical Physics \\ 12 Drahomanov Str., Lviv UA-79005, Ukraine
}

(Received September 7, 2001)

\begin{abstract}
Using the supersymmetric method the quasi-exactly solvable (QES) periodic potentials with two known eigenstates have been constructed. The explicit examples of QES periodic potentials are presented.
\end{abstract}

Key words: supersymmetry, quantum mechanics, quasi-exactly solvable periodic potentials.

PACS number(s): 03.65.Ge, 11.30.Pb

\section{INTRODUCTION}

The energy spectrum of the electrons on a lattice as a central problem of condensed matter physics has been investigated for a long time. Nevertheless, there is rather a limited number of exactly solvable periodic potentials even in one dimension. As the classical text-book example of the exactly solvable potential one can mention the Kronig-Penny model. Another well-studied class of periodic potentials is the class of the elliptic function potential or Lamés potential [1].

Because of the fact that the number of the exactly solvable potentials is limited, much attention has been given recently to the quasi-exactly solvable (QES) potentials for which a finite number of the energy levels and the corresponding wave functions are known explicitly. The class of QES trigonometric periodic potentials was presented in [2]. In [3] it was shown that the Lamé equation is a peculiar example of QES systems. The authors of paper [4] considered a family of spectral equation which extends those of [3]. A short time ago some new exactly solvable and QES periodic potentials were discovered [5$11]$.

The powerful tool for studying the problem of exact solvability of the Schrödinger equation is the supersymmetric (SUSY) quantum mechanics (for a review of SUSY quantum mechanics see [12]). Various aspects of SUSY quantum mechanics with periodic potentials have been discussed in $[5,6]$. In [7-9] using the formalism of SUSY quantum mechanics a large number of new solvable and QES periodic potentials was obtained. Note also a very recent paper [10] where the second-order SUSY transformation was applied for studying periodic potentials.

In our recent works $[13,14]$ we have proposed a new SUSY method for the construction of the QES potentials with two known eigenstates. Within the frame of this method using nonsingular superpotentials we obtained QES potentials for which we found in the explicit form the energy levels and the wave functions of the ground and first excited states. In [15] we have extended this SUSY method for constructing QES potentials with arbitrary two known eigenstates using singular superpo- tentials. Note, that paper [15] was inspired by paper by Dolya and Zaslavskii [16] where they showed how to generate QES potentials with arbitrary two known eigenstates without resorting to the SUSY quantum mechanics.

In this paper using the results of our previous study [15] we generate the QES periodic potentials with two known eigenstates.

\section{SUSY QUANTUM MECHANICS AND QES PROBLEMS}

In this section we reproduce the main results of our previous papers [13-15] and adapt them for the generating QES periodic potentials.

In the Witten's model of supersymmetric quantum mechanics the SUSY partner Hamiltonians $H_{ \pm}$read

$$
H_{ \pm}=B^{\mp} B^{ \pm}=-\frac{1}{2} \frac{d^{2}}{d x^{2}}+V_{ \pm}(x)
$$

where

$$
\begin{aligned}
& B^{ \pm}=\frac{1}{\sqrt{2}}\left(\mp \frac{d}{d x}+W(x)\right), \\
& V_{ \pm}(x)=\frac{1}{2}\left(W^{2}(x) \pm W^{\prime}(x)\right), W^{\prime}(x)=\frac{d W(x)}{d x}
\end{aligned}
$$

and $W(x)$ is referred to as a superpotential. In this paper we shall consider the systems on the full real line $-\infty<x<\infty$ with periodic superpotential $W(x+L)=$ $W(x)$. This superpotential leads to the periodic potential energy $V_{ \pm}(x+L)=V_{ \pm}(x)$.

We shall study the eigenvalue problem for the Hamiltonian $H_{-}$

$$
B^{+} B^{-} \psi_{E}^{-}(x)=E \psi_{E}^{-}(x)
$$

It is easy to show that the eigenfunctions of $H_{-}$and $H_{+}$ are related as follows 


$$
\begin{aligned}
& \psi_{E}^{+}(x)=C B^{-} \psi_{E}^{-}(x), \\
& \psi_{E}^{-}(x)=C B^{+} \psi_{E}^{+}(x) .
\end{aligned}
$$

Due to the factorization of the Hamiltonian the wave function of the zero energy state for $H_{-}$satisfies the equation $B^{-} \psi_{0}^{-}=0$ and reads

$$
\psi_{0}^{-}(x)=C_{0}^{-} \exp \left(-\int W(x) d x\right),
$$

where $C_{0}^{-}$is the constant.

For the periodic systems the wave eigenfunctions are the Bloch functions. Then for the zero energy ground state they must be periodic functions with the same period $L$ as the potential energy. The superpotential in this case is a periodic function and must satisfy the condition $[5,6]$

$$
\int_{0}^{L} W(x)=0
$$

which leads to a restriction of the wave function. In $[5,6]$ a detailed analysis of the SUSY quantum mechanics was done for this case.

In order to obtain one more state of $H_{-}$we use the following well-known procedure exploited in the frame of SUSY quantum mechanics. Let us consider the SUSY partner of $H_{-}$, i. e., the Hamiltonian $H_{+}$. If we calculate some state of $H_{+}$we immediately find a new excited state of $H_{-}$using transformation (6). In order to calculate some state of $H_{+}$let us rewrite it in the following form

$$
H_{+}=H_{-}^{(1)}+\epsilon=B_{1}^{+} B_{1}^{-}+\epsilon, \epsilon>0
$$

which leads to the following relation between the potential energies and superpotentials

$$
\begin{array}{r}
V_{+}(x)=V_{-}^{(1)}(x)+\epsilon, \\
W^{2}(x)+W^{\prime}(x)=W_{1}^{2}(x)-W_{1}^{\prime}(x)+2 \epsilon,
\end{array}
$$

where $\epsilon$ is the energy of the state of $H_{+}$since we supposed that $H_{-}^{(1)}$ similarly to $H_{-}$has the zero-energy state, $B_{1}^{ \pm}$ and $V_{-}^{(1)}(x)$ are given by $(2)$ and $(3)$ with the new superpotential $W_{1}(x)$.

As we see from (9) the wave function of $H_{+}$with the energy $E=\epsilon$ being also the zero-energy wave function of $H_{-}^{(1)}$ and satisfying the equation $B_{1}^{-} \psi_{\epsilon}^{+}(x)=0$. The solution of this equation is

$$
\psi_{\epsilon}^{+}(x)=C^{+} \exp \left(-\int W_{1}(x) d x\right)
$$

Using (6) we obtain the wave function of the excited state with the energy level $E=\epsilon$ for the Hamiltonian $H_{-}$

$$
\psi_{\epsilon}^{-}(x)=C^{-} W_{+}(x) \exp \left(-\int W_{1}(x) d x\right),
$$

where we have introduced the notation $W_{+}(x)=$ $W_{1}(x)+W(x)$

In order to obtain an explicit expression for the wave functions $\psi_{0}^{-}(x)$ and $\psi_{\epsilon}^{-}(x)$ given by $(7)$ and (13) it is necessary to obtain an explicit expression for superpotentials $W(x)$ and $W_{1}(x)$. The superpotentials $W(x)$ and $W_{1}(x)$ satisfy equation (11). It is the Riccati equation which cannot be solved exactly with respect to $W_{1}(x)$ for the given $W(x)$ and vice versa. But we can find such a pair of $W(x)$ and $W_{1}(x)$ that satisfies equation (11). For this purpose let us rewrite equation (11) in the following form

$$
W_{+}^{\prime}(x)=W_{-}(x) W_{+}(x)+2 \epsilon,
$$

where

$$
\begin{aligned}
& W_{+}(x)=W_{1}(x)+W(x), \\
& W_{-}(x)=W_{1}(x)-W(x) .
\end{aligned}
$$

We can solve this new equation with respect to $W_{-}(x)$ for the given $W_{+}(x)$ as well as with respect to $W_{+}(x)$ for the given $W_{-}(x)$. In this paper we use the solution of equation (14) with respect to $W_{-}(x)$ [13]

$$
W_{-}(x)=\left(W_{+}^{\prime}(x)-2 \epsilon\right) / W_{+}(x) .
$$

Then from (15), (16) and (17) we obtain the pair of $W(x), W_{1}(x)$ that satisfies equation (11)

$$
\begin{gathered}
W(x)=\frac{1}{2}\left(W_{+}(x)-\left(W_{+}^{\prime}(x)-2 \epsilon\right) / W_{+}(x)\right), \\
W_{1}(x)=\frac{1}{2}\left(W_{+}(x)+\left(W_{+}^{\prime}(x)-2 \epsilon\right) / W_{+}(x)\right),
\end{gathered}
$$

here $W_{+}(x)$ is some function of $x$ which generates the superpotentials $W(x)$ and $W_{1}(x)$. It is necessary to note that the general solutions (18), (19) of equation (11) were obtained earlier in [17] in the context of parasupersymmetric quantum mechanics.

In our earlier paper [13] we considered only nonsingular superpotentials $W(x)$ and $W_{1}(x)$. In [15] we showed that it is possible to obtain the nonsingular potential energy using generating function $W_{+}(x)$ with many zeros and singularities as well. In this paper we apply the result obtained in [15] for the periodic generating functions.

Case 1. Suppose that $W_{+}(x)$ has simple zeros at the points $x_{k}$

$$
W_{+}(x)=W_{+}^{\prime}\left(x_{k}\right)\left(x-x_{k}\right)+\frac{1}{2} W_{+}^{\prime \prime}\left(x_{k}\right)\left(x-x_{k}\right)^{2}
$$




\section{M. TKACHUK, O. VOZNYAK}

$$
+O\left(\left(x-x_{k}\right)^{3}\right) .
$$

The zeros of the function $W_{+}(x)$ lead to the poles of the function $W_{-}(x)$ and to the superpotential $W(x)$ with the following behaviour in the vicinity of $x_{k}$

$$
\begin{aligned}
W(x) & =-\left(\frac{1}{2}-\frac{\epsilon}{W_{+}^{\prime}\left(x_{k}\right)}\right) \frac{1}{x-x_{k}} \\
& -\frac{1}{2} \frac{W^{\prime \prime}\left(x_{k}\right)}{W^{\prime}\left(x_{k}\right)}\left(\frac{1}{2}+\frac{\epsilon}{W_{+}^{\prime}\left(x_{k}\right)}\right)+O\left(x-x_{k}\right) .
\end{aligned}
$$

The behaviour of the superpotential $W_{1}(x)$ in the vicinity of $x_{k}$ is similar to $W(x)$ having the opposite sign. This superpotential leads to the following behaviour of the potential energy in the vicinity of $x_{k}$

$$
\begin{aligned}
2 V_{-}(x) & =\left[\left(\frac{\epsilon}{W_{+}^{\prime}\left(x_{k}\right)}\right)^{2}-\frac{1}{4}\right] \\
& \times\left(\frac{1}{\left(x-x_{k}\right)^{2}}-\frac{W^{\prime \prime}\left(x_{k}\right)}{W^{\prime}\left(x_{k}\right)} \frac{1}{\left(x-x_{k}\right)}\right)+O \text { (const). }
\end{aligned}
$$

Thus, in the case

$$
W_{+}^{\prime}\left(x_{k}\right)= \pm 2 \epsilon
$$

the potential energy $V_{-}(x)$ is free of singularities. It is convenient to split the set of $x_{k}$ into two subsets $x_{k}^{+}$ and $x_{k}^{-}$for which $W_{+}^{\prime}\left(x_{k}^{+}\right)=2 \epsilon>0$ and $W_{+}^{\prime}\left(x_{k}^{-}\right)=$ $-2 \epsilon<0$. We suppose in this paper that $\epsilon>0$. Because of $W_{+}^{\prime}\left(x_{k}^{+}\right)=2 \epsilon$ the singularity at the points $x_{k}^{+}$is cancelled and $W(x), W_{1}(x)$ have singularities only at the points $x_{k}^{-}$

$$
\begin{gathered}
W(x)=\frac{-1}{x-x_{k}^{-}}+O\left(x-x_{k}^{-}\right), \\
W_{1}(x)=\frac{1}{x-x_{k}^{-}}+O\left(x-x_{k}^{-}\right) .
\end{gathered}
$$

Substituting $W(x)$ into (7) one can show (for details see [15]) that the wave function $\psi_{0}^{-}(x)$ with the zero energy having zeros at the points $x_{k}^{-}$, namely, $\psi_{0}^{-} \sim\left(x-x_{k}^{-}\right)$ in the vicinity of $x_{k}^{-}$. Substituting $W_{+}(x)$ given by (20) and $W_{1}(x)$ given by (25) into (13) we find that the wave function $\psi_{\epsilon}^{-}(x)$ with the energy $\epsilon$ has zeros at the points $x_{k}^{+}: \psi_{\epsilon}^{-}(x) \sim\left(x-x_{k}^{+}\right)$.

Case 2. Let us assume that the function $W_{+}(x)$ in addition to the zeros has the simple poles at the points $x_{k}^{0}$ with the behaviour in the vicinity of $x_{k}^{0}$ as follows

$$
W_{+}(x)=\frac{G_{-1}}{x-x_{k}^{0}}+G_{0}+O\left(x-x_{k}^{0}\right) .
$$

Then

$$
\begin{aligned}
& W(x)=\frac{1}{2} \frac{G_{-1}+1}{x-x_{k}^{0}}+\frac{1}{2} \frac{G_{0}}{G_{-1}}\left(G_{-1}-1\right)+O\left(x-x_{k}^{0}\right), \\
& W_{1}(x)=\frac{1}{2} \frac{G_{-1}-1}{x-x_{k}^{0}}+\frac{1}{2} \frac{G_{0}}{G_{-1}}\left(G_{-1}+1\right)+O\left(x-x_{k}^{0}\right) .
\end{aligned}
$$

The superpotential $W(x)(27)$ gives a nonsingular potential energy for the case 2a: $G_{-1}=-1$ and $G_{0}$ is an arbitrary constant, and for the case $2 b: G_{-1}=-3$ and $G_{0}=0$.

Case $2 a$. For the case $G_{-1}=-1$ in the vicinity of $x_{k}^{0}$ we have the nonsingular superpotential $W(x)$ and the singular one $W_{1}(x)$

$$
\begin{aligned}
& W(x)=G_{0}+O\left(x-x_{k}^{0}\right), \\
& W_{1}(x)=\frac{-1}{x-x_{k}^{0}}+O\left(x-x_{k}^{0}\right) .
\end{aligned}
$$

The wave functions $\psi_{0}^{-}(x)$ and $\psi_{\epsilon}^{-}(x)$ calculated with these superpotentials do not have zeros at the points $x_{k}^{0}$, which we shall denote in this case as $a_{k}$. Using the result obtained in case 1 we see that $\psi_{0}^{-}(x)$ has zeros at $x_{k}^{-}$and $\psi_{\epsilon}^{-}$has zeros at $x_{k}^{+}$.

Case 2b. The case $G_{-1}=-3$ and $G_{0}=0$ leads to the following behaviour of superpotentials in the vicinity of $x_{k}^{0}$

$$
\begin{aligned}
& W(x)=\frac{-1}{x-x_{k}^{0}}+O\left(x-x_{k}^{0}\right), \\
& W_{1}(x)=\frac{-2}{x-x_{k}^{0}}+O\left(x-x_{k}^{0}\right) .
\end{aligned}
$$

The wave functions $\psi_{0}^{-}(x)$ and $\psi_{\epsilon}^{-}(x)$ calculated with these superpotentials have common zeros at the points $x_{k}^{0}$, which we shall denote in this case as $b_{k}$. Thus, when in addition to poles the function $W_{+}(x)$ has zeros at the 
points $x_{k}^{+}, x_{k}^{-}$, the wave function $\psi_{0}^{-}(x)$ has zeros at $x_{k}^{0}$, $x_{k}^{-}$and $\psi_{\epsilon}^{-}(x)$ has zeros at $x_{k}^{0}, x_{k}^{+}$.

Let us consider a general case which combines the cases $1,2 \mathrm{a}$ and $2 \mathrm{~b}$. The function $W_{+}(x)$ has zeros with negative derivatives at the points $x_{k}^{-}$, poles at the points $a_{k}$ with asymptotic behaviour in the vicinity of these points $-1 /\left(x-a_{k}\right)+$ const and poles at the points $b_{k}$ with the asymptotic behaviour $-3 /\left(x-b_{k}\right)$. The wave function $\psi_{0}^{-}(x)$ has the nodes at the points $x_{k}^{-}$and $b_{k}$. The wave function $\psi_{\epsilon}^{-}(x)$ has the nodes at the points $x_{k}^{+}$and $b_{k}$.

Thus, the considered cases $1,2 \mathrm{a}, 2 \mathrm{~b}$ and the combined general case lead to the nonsingular QES periodic potential energy $V_{-}(x)$ given by $(3)$, where the superpotential $W(x)$ is expressed over the function $W_{+}(x)$ by equation (18). The zero-energy wave function $\psi_{0}^{-}(x)$ and the wave function $\psi_{\epsilon}^{-}(x)$ with the energy $\epsilon$ are given by (7) and (13), respectively. In order to obtain the bounded wave functions the superpotentials $W(x)$ and $W_{1}(x)$ must satisfy condition (8). One of the easiest ways to satisfy condition $(8)$ for $W(x)$ and $W_{1}(x)$ is to choose the odd periodic functions $W_{+}(x)$. Then as one can see from (18), (19) the superpotentials $W(x)$ and $W_{1}(x)$ are the odd periodic functions too and thus they satisfy condition (8).

Note, that in the case of nonsingular superpotential $W(x)$ the zero energy wave function corresponds to the ground state, but in the case of singular superpotential the energy of the ground state is less than zero and the zero-energy wave function corresponds to an excited state.

In conclusion of this section let us recall that a solution of the Schrödinger equation with periodic potential satisfies the condition

$$
\psi(x+L)=e^{i k L} \psi(x)
$$

where $k$ is called the quasi-momentum or the momentum of the crystal. The energy spectrum for the periodic potential has the band structure, i. e., the eigenvalues belong to the allowed energy bands $\left[E_{0}, E_{1}\right],\left[E_{1}, E_{2}\right], \ldots$. The edges of the band correspond to $k L=0, \pi$ and the wave functions at the band edges satisfy the condition $\psi(x+L)= \pm \psi(x)$. Note that the band edge energies and the wave functions are often called eigenvalues and eigenfunctions. The oscillation theorem states that for a periodic potential with period $L$ the band edge wave function arranged in order of increasing energy $E_{0} \leq E_{1} \leq E_{1^{\prime}} \leq E_{2} \leq E_{2^{\prime}} \leq E_{3} \ldots$ are of period $\bar{L}, 2 L, 2 \bar{L}, L, L, 2 \bar{L}, 2 L, \ldots$. The corresponding numbers of wave functions nodes in the interval $L$ are $0,1,1,2,2,3,3, \ldots$.

\section{EXAMPLES OF QES PERIODIC POTENTIALS}

Note, that all expressions depend on the generating function $W_{+}(x)$. We may choose various functions $W_{+}(x)$ and obtain as a result various QES potentials. To illustrate the above described method we give few explicit examples of the nonsingular QES periodic potentials.

\section{Example 1}

Let us consider a continuous function $W_{+}(x)$ which corresponds to the case 1

$$
W_{+}(x)=\alpha \sin x
$$

where $\alpha>0$. This function has zeros with positive derivatives at the points $x_{k}^{+}=2 \pi k$ and negative derivatives at $x_{k}^{-}=\pi(2 k+1)$. From the condition of nonsingularity of the potential energy it follows that $\epsilon=$ $W^{\prime}(2 \pi k) / 2=\alpha / 2$. Then using (18) and (19) we obtain for the superpotentials

$$
\begin{aligned}
& W(x)=\frac{1}{2}\left(\alpha \sin x+\tan \frac{x}{2}\right), \\
& W_{1}(x)=\frac{1}{2}\left(\alpha \sin x-\tan \frac{x}{2}\right) .
\end{aligned}
$$

The superpotential $W(x)$ gives the following QES potential

$$
16 V_{-}(x)=\alpha(\alpha+4)-2-\alpha^{2} \cos 2 x-8 \alpha \cos x,
$$

which is of the period $L=2 \pi$.

The zero-energy wave function $(7)$ and wave function with the energy $\epsilon=\alpha / 2$ (13) read

$$
\begin{aligned}
& \psi_{0}^{-}(x)=C_{0} \cos \frac{x}{2} \exp \left(\frac{\alpha}{2} \cos x\right), \\
& \psi_{\epsilon}^{-}(x)=C_{\epsilon} \sin \frac{x}{2} \exp \left(\frac{\alpha}{2} \cos x\right) .
\end{aligned}
$$

As we see $\psi_{0}^{-}(x)$ is of period $2 L=4 \pi$, has one node at the interval $L$ and $\psi_{0}^{-}(x+2 \pi)=-\psi_{0}^{-}(x)$. The wave function $\psi_{\epsilon}^{-}(x)$ is of the period $2 L=4 \pi$, has one node at the interval $L$ and $\psi_{\epsilon}^{-}(x+2 \pi)=-\psi_{\epsilon}^{-}(x)$. The function $\psi_{0}^{-}(x)$ corresponds to the energy $E_{1}$ which is the upper edge energy of the first band. The function $\psi_{\epsilon}^{-}(x)$ corresponds to the energy $E_{1}$, which is the lower edge energy of the second band. Thus, the gap between the first and second bands is equal to $E_{1},-E_{1}=\epsilon=\alpha / 2$. Note, that this example reproduces one of the potentials studied in $[2]$.

\section{Example 2}

Let us consider a more complicated example for which the function $W_{+}(x)$ has zeros and poles. One of the simplest functions of this kind is as follows

$$
W_{+}(x)=\alpha \sin (2 x)+\tan x=\left(1+2 \alpha \cos ^{2} x\right) \tan x
$$

Let $\alpha>-1 / 2$. Then zeros and poles of $W_{+}(x)$ originate from $\tan x$ only. Note that this generating function 


\section{M. TKACHUK, O. VOZNYAK}

corresponds to case 2 a.

Choosing

$$
\epsilon=\frac{W_{+}^{\prime}(0)}{2}=\alpha+\frac{1}{2}
$$

we obtain the superpotentials

$$
\begin{aligned}
& W(x)=\frac{4+\alpha+\alpha \cos 2 x}{1+\alpha+\alpha \cos 2 x} \frac{\alpha}{2} \sin 2 x, \\
& W_{1}(x)=\frac{2 \alpha^{2} \cos ^{3} x \sin x+\tan x}{1+\alpha+\alpha \cos 2 x}
\end{aligned}
$$

and the nonsingular potential energy

$$
\begin{aligned}
16 V_{-}(x) & =-6+\alpha(12+\alpha)-20 \alpha \cos 2 x-\alpha^{2} \cos 4 x \\
& +\frac{6+24 \alpha^{2} \cos ^{2} x}{(1+\alpha+\alpha \cos 2 x)^{2}}
\end{aligned}
$$

The potential energy $V_{-}(x)$ is of the period $L=\pi$. For this QES potential we know explicitly the wave functions which correspond to the zero energy and the energy $\epsilon=\alpha+1 / 2$, respectively,

$$
\begin{aligned}
& \psi_{0}^{-}(x)=C_{0}(1+\alpha+\alpha \cos 2 x)^{3 / 4} \exp \left(\frac{1}{4} \alpha \cos 2 x\right), \\
& \psi_{\epsilon}^{-}(x)=C_{\epsilon}(1+\alpha+\alpha \cos 2 x)^{1 / 4} \sin x \exp \left(\frac{1}{4} \alpha \cos 2 x\right) .
\end{aligned}
$$

As we see $\psi_{0}^{-}(x)$ is of the period of the potential energy $L=\pi$, has no nodes and corresponds to the ground state. The wave function $\psi_{\epsilon}^{-}(x)$ is of the period $2 L$, has one node at the interval $L=\pi$ and in addition satisfies $\psi_{\epsilon}^{-}(x+\pi)=-\psi_{\epsilon}^{-}(x)$. This function can correspond to the upper edge energy $E_{1}$ of the first band or to the lower edge energy $E_{1}$, of the second band. In order to find to which band the wave function $\psi_{\epsilon}^{-}(x)$ belongs we consider the system on a circle. Let us assume that the number of the elementary cells on the circle is $n$. Then the allowed band contains $n$ levels. In the considered case the number of zeros of $W_{+}(x)$ at $x_{k}^{+}$is the same as the number of poles at $x_{k}^{0}$ and is equal to $n$. Then according to case 2a $\psi_{\epsilon}^{-}(x)$ has nodes at $x_{k}^{+}$the number of which is equal to $n$. Thus, this function corresponds to the upper edge energy $E_{1}$ of the first band. It is interesting to note that for $\alpha=0$ we arrive at the free particle case.

\section{Example 3.}

In this example we consider the generating function which corresponds to the case $2 \mathrm{~b}$

$$
\begin{aligned}
W_{+}(x) & =\alpha \sin (2 x)+3 \tan x \\
& =\left(3+2 \alpha \cos ^{2} x\right) \tan x .
\end{aligned}
$$

Let $\alpha>-3 / 2$. Then like in the example 2 the zeros and poles of $W_{+}(x)$ originate from tan $x$ only. Choosing

$$
\epsilon=\frac{W_{+}^{\prime}(0)}{2}=\alpha+\frac{3}{2}
$$

we obtain the superpotentials

$$
\begin{aligned}
& W(x)=\frac{\alpha}{2} \sin 2 x+\tan x+\frac{3 \alpha \sin 2 x}{2(3+\alpha+\alpha \cos 2 x)}, \\
& W_{1}(x)=2 \tan x+\frac{2 \alpha^{2} \sin x \cos ^{3} x}{3+\alpha+\alpha \cos 2 x}
\end{aligned}
$$

and the nonsingular potential energy

$$
\begin{aligned}
16 V_{-}(x) & =-14+\alpha(20+\alpha)-28 \alpha \cos 2 x \\
& -\alpha^{2} \cos 4 x+\frac{54-24 \alpha^{2} \cos ^{2} x}{(3+\alpha+\alpha \cos 2 x)^{2}},
\end{aligned}
$$

which is of the period $L=\pi$. For this QES potential we know explicitly the wave functions which correspond to the zero energy and the energy $\epsilon=\alpha+3 / 2$, respectively,

$$
\begin{aligned}
& \psi_{0}^{-}(x)=C_{0}(3+\alpha+\alpha \cos 2 x)^{3 / 4} \cos x \exp \left(\frac{1}{4} \alpha \cos 2 x\right), \\
& \psi_{\epsilon}^{-}(x)=C_{\epsilon}(3+\alpha+\alpha \cos 2 x)^{1 / 4} \sin 2 x \exp \left(\frac{1}{4} \alpha \cos 2 x\right) .
\end{aligned}
$$


The function $\psi_{0}^{-}(x)$ is of the period $2 L=2 \pi$, has one node at the interval $L=\pi$ and satisfies the condition $\psi_{0}^{-}(x+\pi)=-\psi_{0}^{-}(x)$. The function $\psi_{\epsilon}^{-}(x)$ is of the period $L=\pi$, has two nodes at the interval $L=\pi$ and satisfies the condition $\psi_{\epsilon}^{-}(x+\pi)=\psi_{\epsilon}^{-}(x)$. In order to find to which energy edge bands these functions correspond we consider our system on the circle similarly as for example 2. Then the wave function $\psi_{0}(x)$ has nodes at $x_{k}^{0}$ the number of which is $n$ and thus it corresponds to the upper energy edge $E_{1}$ of the first band. The wave function $\psi_{\epsilon}^{-}(x)$ has the nodes at the points $x_{k}^{0}$ and $x_{k}^{+}$ the number of which is $2 n$ and thus this function corresponds to the upper energy edge $E_{2}$ of the second band. Note, that similarly to example 2 in this case for $\alpha=0$ we have a free particle.

\section{CONCLUSIONS}

In this paper we have extended the SUSY method of construction well-like QES potentials proposed in our earlier papers [13-15] for the case of periodic potentials. Choosing different periodic generating functions $W_{+}(x)$ we have obtained different QES periodic potentials $V_{-}(x)$ for which we know in the explicit form two eigenstates. Example 1 reproduces one of the potentials studied in [2]. Examples 2 and 3 yield some new QES periodic potentials. It is interesting to note that at the value of the parameter $\alpha=0$ these examples reduce to the case of a free particle.
[1] F. M. Arscott, Periodic differential Equation (Pergamon, Oxford, 1981).

[2] A. V. Turbiner, Zh. Eksp. Teor. Fiz. 94, 33 (1988).

[3] A. V. Turbiner, J. Phys. A 22, L1 (1989).

[4] Y. Brihaye, M. Godart, J. Math. Phys. 34, 5283 (1993).

[5] G. Dunne, J. Feinberg, Phys. Rev. D 57, 1271 (1998).

[6] G. Dunne, J. Mannix, Phys. Lett. B 428, 115 (1998).

[7] A. Khare, U. Sukhatme, J. Math. Phys. 40, 5473 (1999).

[8] U. Sukhatme, A. Khare, preprint quant-ph/9902072 (1999).

[9] A. Khare, U. Sukhatme, J. Math. Phys. 42, 5652 (2001); preprint quant-ph/0105044 (2001).
[10] D. J. Fernández C., J. Negro, L. M. Nieto, Phys. Lett. A 275, 338 (2000).

[11] A. Khare, Phys. Lett. A 288, 69 (2001); preprint quantph/0105030 (2001).

[12] F. Cooper, A. Khare, U. Sukhatme, Phys. Rep. 251, 267 (1995).

[13] V. M. Tkachuk, Phys. Lett. A 245, 177 (1998).

[14] V. M. Tkachuk, J. Phys. A 32, 1291 (1999).

[15] V. M. Tkachuk, J. Phys. A 34, 6339 (2001).

[16] S. N. Dolya, O. V. Zaslavskii, J. Phys. A 34, 1981 (2001).

[17] J. Beckers, N. Debergh, A. G. Nikitin, Mod. Phys. Lett. A 8, 435 (1993).

\title{
СУПЕРСИМЕТРИЧНИЙ ПІДХІД ДЛЯ ІЕНЕРАЦІЇ КВАЗІТОЧНО РОЗВ'ЯЗУВАНИХ ПЕРІОДИЧНИХ ПОТЕНЦЯЛІВ
}

\author{
В. М. Ткачук, О. Возняк \\ Львівський наиіональний університет імені Ібана Франка, кафедра теоретичной фізики, \\ вул. Драгоманова, 12, Львів, 79005, Украӥна \\ e-mail: tkachuk@ktf.franko.lviv.ua
}

Із використанням методу суперсиметрії отримано квазіточно розв'язувані потенціяли з двома відомими власними станами. Наведено явні приклади квазіточно розв'язуваних потенщіялів. 